\title{
Identificación de factores que afectan la aplicación de la prueba Short Physical Performance Battery en centros de atención para el adulto mayor
}

\author{
Identification of affecting factors for the \\ SPPB test in senior attention centers
}

\author{
María Cristina Vargas-Del-Valle ${ }^{1}$, Olga Sánchez-Brenes², \\ Magaly Sánchez-Brenes ${ }^{3}$, Arturo Huete-Calderón ${ }^{4}$, Ronny Arias-Mora ${ }^{5}$
}

Fecha de recepción: 9 de julio de 2020

Fecha de aprobación: 18 de octubre de 2020

Vargas-Del-Valle, M.C; Sánchez-Brenes, O; Sánchez-Brenes, M; Huete-Calderón, A; Arias-Mora, R. Identificación de factores que afectan la aplicación de la prueba Short Physical Performance Battery en centros de atención para el adulto mayor. Tecnología en Marcha. Vol. 34-4 Octubre-Diciembre 2021. Pág 192-203.

doi) https://doi.org/10.18845/tm.v34i4.5204

1 Laboratorio de Ergonomía Aplicada, Instituto Tecnológico de Costa Rica. Costa Rica. Correo electrónico: crisvargasdelvalle@gmail.com

2 Laboratorio de Ergonomía Aplicada, Instituto Tecnológico de Costa Rica. Costa Rica. Correo electrónico: olsanchez@tec.ac.cr

3 Laboratorio de Ergonomía Aplicada, Instituto Tecnológico de Costa Rica. Costa Rica. Correo electrónico: msanchez@tec.ac.cr https://orcid.org/0000-0002-8107-3361

4 Escuela de Ingeniería en Diseño Industrial, Instituto Tecnológico de Costa Rica. Costa Rica. Correo electrónico: arturohuete@hotmail.com https://orcid.org/0000-0003-1351-0117

5 Área académica de Ingeniería Mecatrónica, Instituto Tecnológico de Costa Rica. Costa Rica. Correo electrónico: ronarias.m@gmail.com 


\title{
Palabras clave
}

Adulto mayor; batería funcional; SPPB; desempeño físico.

\section{Resumen}

El objetivo de este estudio es la identificación de factores que influyen en la aplicación de la prueba Short Physical Performance Battery (SPPB) para la valoración funcional en adultos mayores. Esta evaluación caracteriza las causas que afectan la medición y su relación con la fiabilidad de los datos. Los puntajes son determinantes para establecer algún criterio de fragilidad en la persona mayor y con los cuales se establecerán intervenciones adecuadas. Los datos fueron recolectados utilizando un enfoque mixto de observaciones directas de los involucrados, mapeo de procesos y procedimientos de la prueba, entrevistas a especialistas y adultos mayores, revisión de notas de campo, fotografías y video. Los resultados en la aplicación de la prueba muestran tiempos muy similares al mínimo reportado en la teoría, sin embargo, esos tiempos están basados en ambientes controlados por lo que no se contemplan factores que se presentan en la realidad y que afectan directamente la aplicación de la prueba. Además, los elementos con mayor influencia son el tipo de paciente, el instrumento de medición utilizado, el proceso para realizar la prueba y los sistemas de registro utilizado, entre otros. Este estudio brinda información útil acerca de la labor que se realiza en la valoración funcional dentro de la atención geriátrica de Costa Rica. Comprender esta dinámica ayuda a futuras investigaciones para apoyar la labor diaria de los especialistas en salud del país mediante la vinculación entre la ingeniería y la salud, mejorando así la calidad de vida de personas de la tercera edad.

\section{Keywords}

Elderly; functional battery; SPPB; physical performance.

\begin{abstract}
The goal of this study is to identify causes that influence the application of the Short Physical Performance Battery (SPPB), a functional performance test for older adults. This evaluation describes the main causes that affect the measurement and the relationship between them and the reliability of the data. These scores are decisive in establishing some frailty criteria in the elderly and with which the appropriate guidelines and programs will be established. Data was collected using a mixed approach of direct observations, mapping of the procedures, interviews with specialists and older adults, review of field notes, photographs and video. Results in the application of the battery test show times similar to the minimum reported in theory, however, these times were established in controlled environments, leaving out factors that occur in a daily application. In addition, the elements with the greatest influence are the type of patient, the measurement instrument used, the process to perform the test and the registration systems used, among others. This study provides useful information about the work carried out in functional assessment within geriatric care in Costa Rica. Understanding this dynamic helps future research to support the daily work of the health specialists by linking engineering and health, thus improving the quality of life of the elderly.
\end{abstract}

\section{Introducción}

Según la Organización Mundial de la Salud en su Informe Mundial sobre el Envejecimiento y la Salud del 2015, entre el año 2000 y el 2050 la población mayor de 60 años aumentará de 605 a 2000 millones de personas. Además, recalca que "habrá más personas octogenarias y 
nonagenarias que nunca antes". El Instituto Nacional de Estadísticas y Censos (INEC) en sus proyecciones demográficas para el 2045 [1], prevé un aumento en la población mayor (65 años o más) de un 8,5\% en el 2018 a un 18\% en el 2045.

Un factor fundamental en este proceso de envejecimiento es la pérdida de la fuerza muscular y, por ende, la capacidad motora. Este fenómeno inicia a los 30 años y continúa hasta los 80 años con una disminución entre el 30 y el 50\% [2] lo que impacta significativamente la independencia y autonomía del adulto mayor y, por lo tanto, es necesario la incorporación de procesos de prevención y rehabilitación.

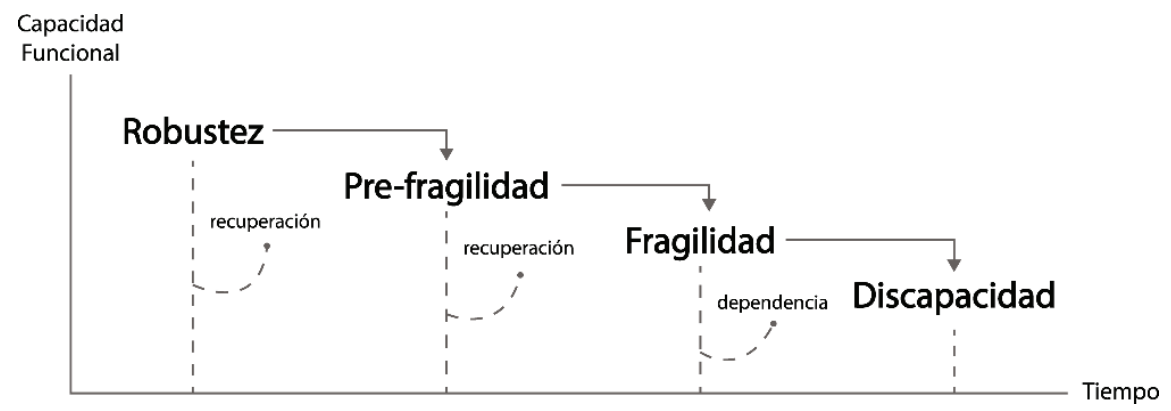

Figura 1. Cascada de disminución funcional en adultos mayores desde la independencia hasta la fragilidad y discapacidad [3].

La fragilidad física en un adulto mayor puede ser considerada como un estado anterior a la discapacidad (o asistencia necesaria en Actividades de la Vida Diaria (AVD)). Sin embargo, no necesariamente una persona mayor tendrá un criterio de fragilidad, ya que existen otras causas que pueden empeorarla como la inactividad física. [3]. Otro aspecto es la sarcopenia o una disminución en la fuerza muscular, la masa muscular (en calidad y en cantidad) y, por lo tanto, el rendimiento físico. Además, la fragilidad está asociada al incremento de caídas, fracturas, discapacidad física y mortalidad [4]. Por ello, es de suma importancia comprender la dinámica del adulto mayor a lo largo del proceso natural de la fragilidad (figura 1). Y, una identificación e intervención adecuada puede revertir, ralentizar o detener el avance de la fragilidad a lo largo de los 4 estados. Esto permite que el adulto mayor tenga un mejor envejecimiento de acuerdo a su condición.

El sistema de salud de Costa Rica ha establecido distintos niveles geriátricos de atención para los adultos mayores. Estos niveles responden a la capacidad de atención de la red de recursos institucionales nacionales desde el ámbito sanitario especializado [5]. El Hospital Dr. Raúl Blanco Cervantes (HBC) está dentro del tercer nivel de atención siendo el centro con mayor especialización geriátrica a nivel nacional; atiende anualmente 9.250 pacientes aplicando hasta 5.061 terapias diferentes, y cuenta con un equipo profesional especializado. Esta institución ofrece una modalidad de atención llamada Hospital de Día (HD), en el cual los adultos mayores son atendidos durante el día para luego regresar a sus casas. El HD brinda un programa de rehabilitación física para pacientes con algún criterio de fragilidad. Los pacientes deben ser evaluados y calificados según su condición física, para luego planificar terapias adecuadas y mejorar su estado. La valoración funcional determina la manera en la que los especialistas enfocarán su plan terapéutico, por lo tanto, medir el desempeño y darles un criterio de fragilidad de la manera correcta es de suma importancia. La prueba que utiliza el HBC para esa valoración funcional es una batería o serie de pruebas cortas que miden variables de equilibrio, fuerza muscular de las piernas y la velocidad de la marcha llamada Short Physical Performance Battery 
(SPPB). Esta prueba asigna por cada una de las variables descritas un puntaje y la suma de estos determina la calificación final de la valoración funcional en una escala de 0 a 12 puntos, donde un puntaje menor a 10 indica que el adulto mayor tiene algún criterio de fragilidad.

La SPPB fue creada para la identificación rápida del estado físico de un adulto mayor por medio del diagnóstico en la funcionalidad de extremidades inferiores. Además, las mediciones del desempeño en cada prueba están fuertemente asociadas a la identificación de la discapacidad motora, y las mismas son predictores independientes de mortalidad (a corto plazo) e institucionalización [6]. Es por ello que la comunidad médica utiliza esta batería de pruebas, de entre muchas otras, como un primer acercamiento para la detección e identificación de la fragilidad.

El proceso general de la atención geriátrica para un adulto mayor tiene varias etapas en donde se realizan pruebas de evaluación, diagnóstico, pronóstico e intervención (figura 2). Si esa persona es clasificada dentro de algún criterio de fragilidad, el objetivo principal de los programas de salud y de los especialistas es su retorno al nivel más alto posible dentro de la cascada de disminución funcional. La valoración del rendimiento físico se encuentra dentro de la evaluación del adulto mayor en la cual se utilizan instrumentos validados de medición funcional geriátrica como la prueba SPPB (figura 3).

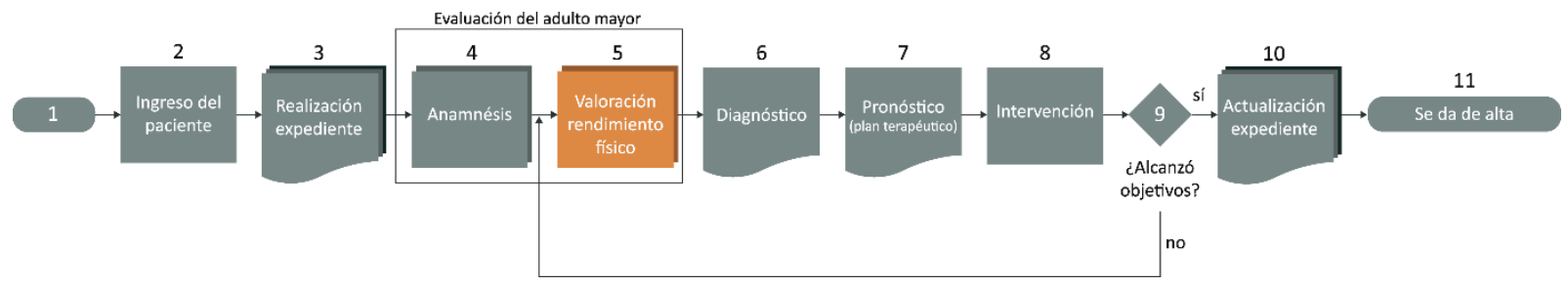

Figura 2. Proceso promedio de la evaluación del adulto mayor en Costa Rica.

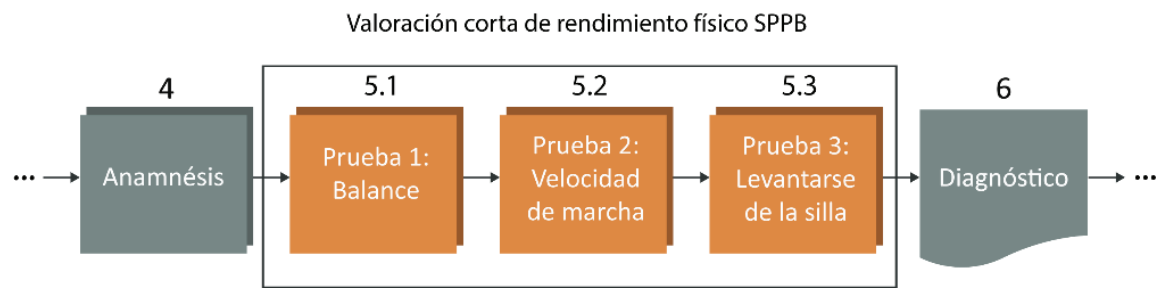

Figura 3. Prueba SPPB dentro del proceso de evaluación del adulto mayor.

\section{Prueba SPPB}

1. Prueba de Equilibrio

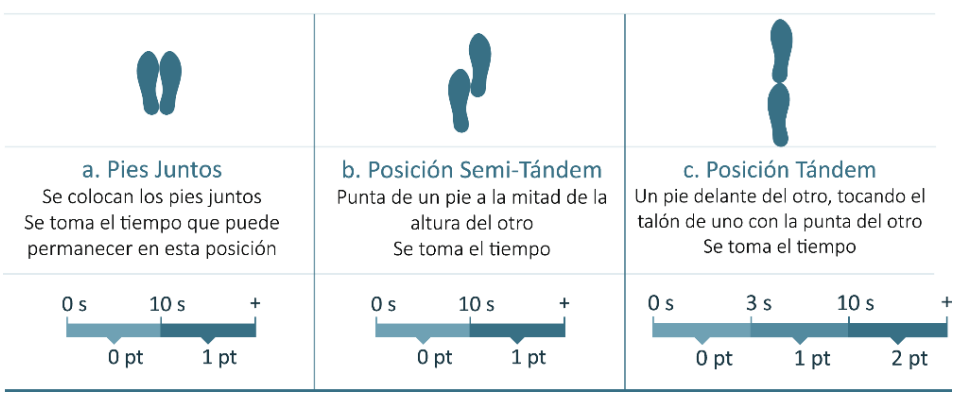

Figura 4. Sub prueba de Balance. Posición paralela, semi-tándem y tándem. 
La primera prueba consiste en medir el equilibrio del adulto mayor por medio del posicionamiento de los pies, durante 10 segundos, en paralelo, tándem y semi tándem (Figura 4). La segunda prueba mide el tiempo que dura el paciente en completar 5 ciclos de levantarse y sentarse de una silla, con el fin de observar la fuerza en el tren inferior (Figura 5). Y la última prueba consiste en medir el tiempo que toma el paciente en completar una distancia de 4 o 6 metros ida y vuelta para conocer la marcha del adulto mayor (Figura 6) [6], [7].

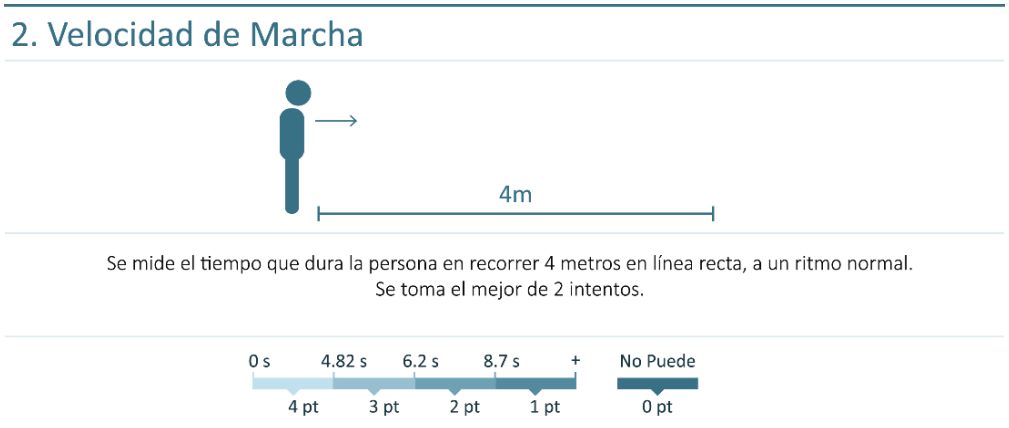

Figura 5. Sub prueba de velocidad de la marcha. Este recorrido se realiza de 2 a 3 veces para sacar un promedio.

3. Levantarse de la Silla

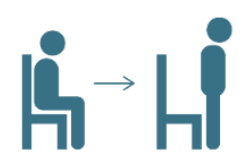

Se ralizan 5 levantamientos lo más rápido posible. Previo a esta prueba se realiza una pre prueba para saber si el adulto mayor puede hacerlo. Los brazos no deben utilizarse de apoyo en la silla.

0s $\begin{array}{rllll}11.2 \mathrm{~s} & 13.7 \mathrm{~s} & 16.7 \mathrm{~s} & 60 \mathrm{~s} & \\ 4 \mathrm{pt} \quad 3 \mathrm{pt} \quad 2 \mathrm{pt} \quad 1 \mathrm{pt} \quad 0 \mathrm{pt}\end{array}$

Figura 6. Sub prueba de levantarse de la silla.

A pesar de que la prueba SPPB tiene una fuerte validación a nivel internacional, es una prueba operador dependiente y en la cual el puntaje final que determina el estado funcional del paciente puede estar influenciado por varios factores asociados a la manera en la que cada especialista aplica la prueba (error humano), los instrumentos de medición que se utilizan, el entorno o los recursos disponibles de tiempo y personal para la aplicación.

Este estudio se enfocó en la identificación de los factores principales que afectan la fiabilidad de los datos en la prueba SPPB, que los especialistas aplican a los adultos mayores para conocer el estado funcional y categorizarlos según su nivel de fragilidad. Para el estudio se contó con la colaboración de especialistas del HBC, así como de la Asociación Cartaginesa de Atención a Ciudadanos de la Tercera Edad (ASCATE). A los participantes se les entregó, leyó y explicó el procedimiento a realizar por medio del consentimiento informado con base en la Ley 9234 (ley reguladora de investigación biomédica) de Costa Rica [8].

Para este estudio se consideraron las normas internacionales para identificar y gestionar la fragilidad en la práctica clínica establecida por la Conferencia Internacional sobre Investigación en Fragilidad y Sarcopenia (ICFSR por sus siglas en inglés). Estas pautas son fuertemente recomendadas por los especialistas debido a los beneficios de la intervención, bajo riesgo 
asociado y mayor aceptación por parte de los pacientes. De las 15 pautas que contiene la investigación, solamente 4 se refieren al estado físico y, por lo tanto, a la prevención en la disminución funcional del adulto mayor. Estas son la detección y la evaluación de la fragilidad, así como la actividad y el ejercicio físico (cuadro 1).

Cuadro 1. Resumen de las recomendaciones de la ICFSR para la identificación y el manejo de la fragilidad en adultos mayores [3].

\begin{tabular}{|c|c|c|}
\hline Recomendación & $\begin{array}{l}\text { Grado de } \\
\text { recomendación }\end{array}$ & $\begin{array}{l}\text { Certeza (calidad) } \\
\text { en la evidencia }\end{array}$ \\
\hline $\begin{array}{l}\text { Detección de la fragilidad } \\
\text { 1. Utilizar un instrumento rápido y validado para detectar la } \\
\text { fragilidad en personas con } 65 \text { o más años. }\end{array}$ & Fuerte & Baja \\
\hline $\begin{array}{c}\text { Evaluación de la fragilidad } \\
\text { 2. La evaluación clínica de la fragilidad debe ser para todos } \\
\text { los adultos mayores, siendo como positiva la pre fragilidad y la } \\
\text { fragilidad. }\end{array}$ & Fuerte & Baja \\
\hline $\begin{array}{l}\text { Actividad/Ejercicio Físico } \\
\text { 6. Se les debe de ofrecer un programa de actividad física } \\
\text { multicomponente (y como prevención). } \\
\text { 7. Se debe referir a personas mayores con debilidad a programas } \\
\text { de entrenamiento en resistencia. }\end{array}$ & Fuerte & Moderada \\
\hline
\end{tabular}

Por medio de la primera recomendación, los especialistas tienen ese acercamiento inicial en la identificación y el manejo de la fragilidad en el adulto mayor. Esos instrumentos rápidos y validados son pruebas que buscan la predicción del estado general de la persona mayor a la hora de la valoración, enfatizado el riesgo de discapacidad y, por lo tanto, la necesidad de admisión a un hogar de ancianos, la dependencia en la atención médica, la disminución continua en las AVD y mortalidad del paciente. La SPPB ha sido una prueba exitosa en predecir el desarrollo de la discapacidad (movilidad reducida, incapacidad de las AVD) y, por lo tanto, se utiliza como instrumento validado a nivel internacional [9].

Sin embargo, la forma en la que se realiza la prueba actualmente puede influir directamente en los resultados de la medición y alterar significativamente el puntaje final con consecuencias en la categorización del adulto mayor en su nivel de fragilidad. Por la escala sensible de la prueba, podría ser fácil incurrir en una puntuación incorrecta o tener un desconocimiento de cuánto cambio, en la puntuación de pruebas consecutivas, se considera significativo para declarar un deterioro físico en el adulto mayor [10].

Las medidas de rendimiento físico son parte de la atención geriátrica y con las cuales se toman decisiones importantes acerca del cuidado necesario para el adulto mayor. Por ello los especialistas no solo requieren de instrumentos validados, sino que los datos obtenidos en la aplicación de las pruebas sean confiables y determinen con certeza el comportamiento a lo largo de la terapia o de su evaluación periódica y si existe alguna alteración en el desempeño del paciente para actuar lo más temprano posible. 


\section{Metodología}

Esta investigación utilizó un enfoque mixto que incluía observaciones directas de los involucrados, mapeo de procesos y procedimientos de pruebas de valoración funcional, entrevistas a especialistas y adultos mayores, y revisión de información específica acerca de la prueba dentro del HBC. Además, se utilizó la metodología de Diseño y desarrollo de producto de Karl T. Ulrich y Steven D. Eppinger. Los capítulos de identificación de oportunidades, identificación de necesidades, así como el de especificaciones de producto se utilizaron como referencia para determinar y analizar los puntos críticos que afectan la aplicación de la prueba SPPB.

Los datos recolectados en la aplicación de la prueba se realizaron de manera observacional con una muestra seleccionada por conveniencia de 5 adultos mayores con diferentes trastornos cognitivos y físicos. Se tomaron los tiempos con un cronómetro de dispositivo móvil mientras la misma fisioterapeuta de ASCATE aplicaba la prueba y tomaba los tiempos con un reloj de pulsera digital. Por cuestiones administrativas, dentro del HBC solo se observó a un adulto mayor sin conocimiento de la edad y con un síndrome de caída, mientras un médico geriatra aplicaba la prueba y se realizaron 2 entrevistas exhaustivas al respecto del procedimiento.

Además, se realizó un mapeo del proceso de la aplicación de la prueba SPPB dentro de una consulta diaria del especialista. La retroalimentación obtenida con las entrevistas, videos y notas de campo suministraron la información necesaria para construir los flujos de trabajo y proceso. Con este insumo y realizando un trabajo iterativo de análisis y observación, se identificaron los factores principales que afectan la fiabilidad de la prueba.

\section{Resultados}

Se procedió a realizar un diagrama de flujo más detallado de cada sub prueba (Figura 7,8,9) para determinar la interacción del especialista con el paciente para la aplicación de la batería de prueba SPPB como parte de la valoración geriátrica. Por medio de este método se identificaron reprocesos y, por lo tanto, puntos críticos que afectan la fiabilidad de los datos y el tiempo de aplicación. Estos reprocesos son dependientes del tipo de paciente al que se le realice la prueba, por lo tanto, se abarcó cada posible escenario en conjunto con los especialistas y apoyados por los videos y observaciones de campo. El proceso se dividió en etapas principales para establecer los inicios y finales de cada tarea. Se midieron los tiempos para cada etapa y se identificó que la mayoría llega a una duración mínima reportada en la teoría de 5 minutos [6], [11]. También se tomó en cuenta el tiempo que tienen los especialistas en cada consulta para realizar la prueba. De acuerdo a las entrevistas, la consulta es de 30 minutos. De aquí la importancia de no sobrepasar los 10 minutos de duración en la aplicación de la prueba de acuerdo al criterio de expertos.

El diagrama de la sub prueba de balance muestra el proceso normal de aplicación en los tonos verdes. A lo largo de las etapas de instrucción en la prueba de equilibrio, un reproceso frecuente es la demostración de las posiciones y el reacomodo del adulto mayor dentro del espacio determinado para mantener el balance. Esto aumenta el tiempo de aplicación pues el especialista debe cerciorase de la posición correcta del paciente para iniciar el cronómetro. En la etapa de medición, el punto más crítico es mantener la seguridad para el adulto mayor y manejar el cronómetro. Esto genera una medición menos precisa influida por el tiempo de reacción del especialista que, a su vez, utiliza los brazos para asegurar la estabilidad de la persona mayor. Para las etapas de registro y ejecución se identificaron situaciones que no son tan críticas pero que aumenta el tiempo de aplicación (cuadro 2). 
Para la sub prueba de velocidad de marcha el mayor momento crítico es el traslado del paciente a un lugar que cumpla con los 4 o 6 metros de distancia para poder realizar el recorrido. Esto causa que la aplicación de la prueba se detenga mientras el paciente vuelve a la oficina del especialista para continuar con la última sub prueba. Además, esta etapa está influida por reprocesos que incluyen la distracción del adulto mayor por terceros mientras se realiza la prueba con lo que se debe reiniciar el cronómetro y la recolocación del material para identificar los 4 o 6 metros pues al ser un espacio común otras personas alteran la marca. Otros factores que aumentan la aplicación de la sub prueba se mencionan en el cuadro 2.

Para la última sub prueba de levantarse de la silla, el punto crítico se ubica en la etapa de medición. Por ser la prueba que demanda mayor esfuerzo para el adulto mayor, es necesario que el especialista realice un paso de verificación para saber si este es capaz de completar la prueba. Este paso implica que el paciente se levante de la silla sin ayuda de los brazos por una vez. Si es capaz de levantarse, se detiene al adulto mayor y se inicia la prueba con la medición. Sin embargo, en algunas ocasiones el adulto mayor continúa levantándose más de una vez en el paso de verificación por lo que el especialista debe detenerlo, explicarle de nuevo e iniciar la medición. Esta confusión provoca que el paciente se agote más rápido con lo que puede perjudicar el tiempo óptimo de su capacidad real. Otros factores que aumentan la aplicación de la sub prueba se mencionan en el cuadro 2. Los factores comunes entre las tres pruebas que no permiten una medición precisa es el tiempo de reacción del especialista y el tipo de instrumento utilizado. El uso del cronómetro de un reloj digital de pulsera o del teléfono celular, aunado a los distintos tiempos de reacción de acuerdo a la manera en la que se usan estos instrumentos desfavorece el puntaje, así como la fiabilidad de los datos.

Los reprocesos generados debido a los puntos críticos de cada etapa aumentan la aplicación de la prueba hasta 15 minutos, dependiendo del tipo de paciente y de las características del espacio en donde se esté realizando. Esta situación obliga a los especialistas a prescindir de la prueba si se encuentran en esta situación, con lo que los pacientes se quedan sin la valoración. En la tabla 2 se muestra el tipo de reproceso en cada etapa y su respectivo el rango de tiempo. Valor que se le sumaría al proceso normal de aplicación. Para el especialista es muy importante valorar el desempeño físico del adulto mayor a través de esta prueba, y la diferencia en sus características físicas y cognitivas influye considerablemente en el tipo de reproceso que se pueda presentar. Por ejemplo, en la prueba de equilibrio los especialistas invierten más tiempo en la etapa de Instrucción cuando después de haber dado la explicación de la posición, los adultos mayores dudan de las indicaciones y el especialista debe demostrar la posición o el recorrido. En este caso, los pacientes con alguna situación cognitiva fueron los que generaron el reproceso. Además, para la etapa de Medición en la misma prueba, un paciente con trastorno cognitivo y otra con patologías a nivel del tronco se perdían en el espacio al realizar la posición semitándem o tándem, lo que ocasionó que el profesional pausara el intento de colocación y los ayudara a tomar la posición. Para la prueba de velocidad, el factor traslado hizo que la prueba se pausara por un tiempo mientras pacientes con andadera o con lesiones en la cadera llegaran. 
Subprueba de Balance (A)

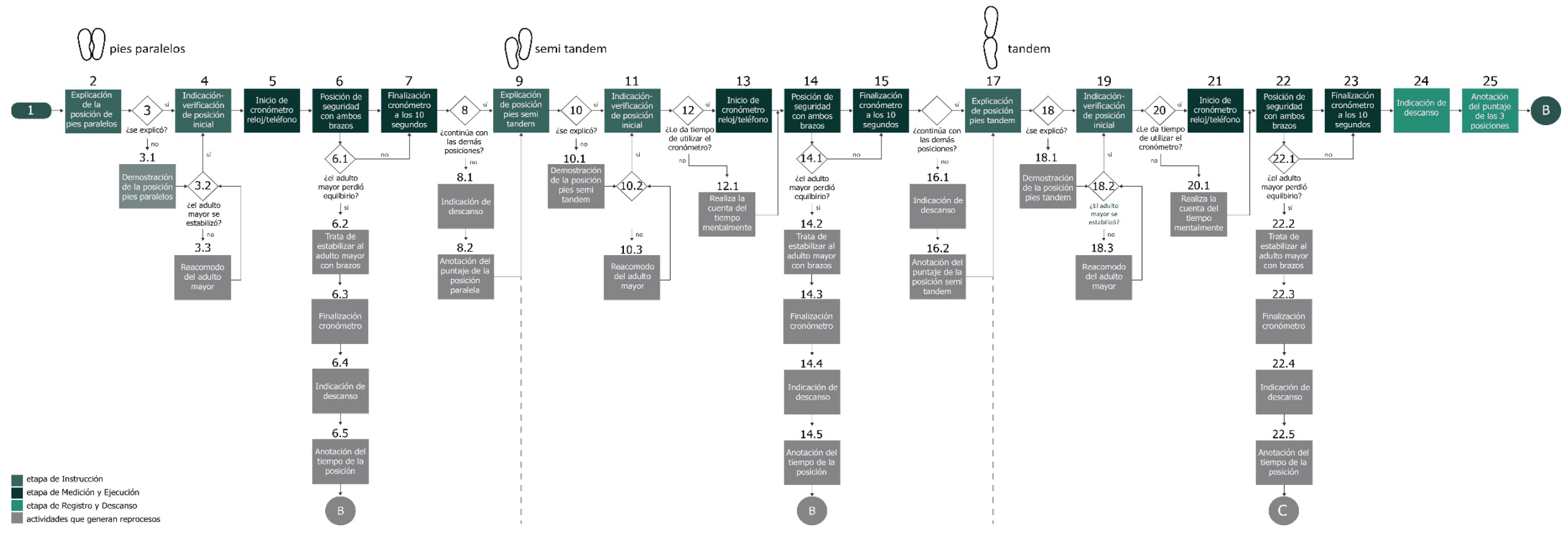

Figura 7. Diagrama de flujo de la prueba de balance (A) dentro de la SPPB.

Subprueba de Velocidad de marcha (B)

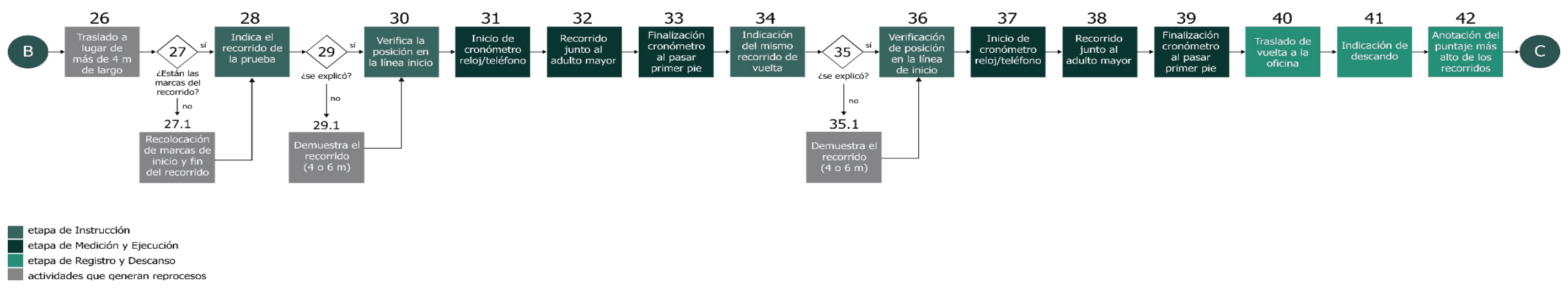

Figura 8. Diagrama de flujo de la prueba de velocidad de marcha (B) dentro de la SPPB. 


\section{Subprueba de Levantarse de la Silla (C)}

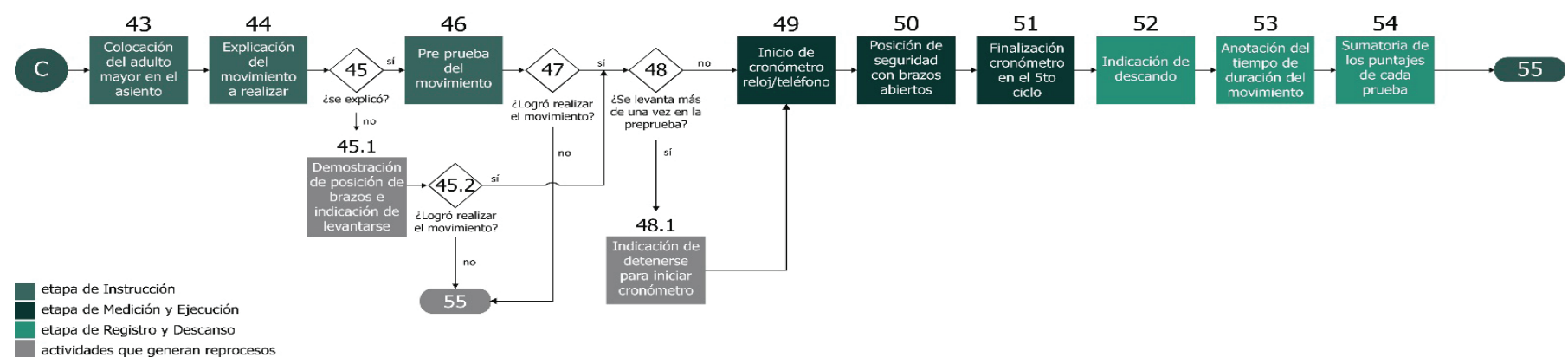

Figura 9. Diagrama de flujo de la prueba de levantarse de la silla (C) dentro de la SPPB.

Cuadro 2. Puntos críticos y rangos de tiempos de reproceso que se le agregan a cada sub prueba en cada etapa.

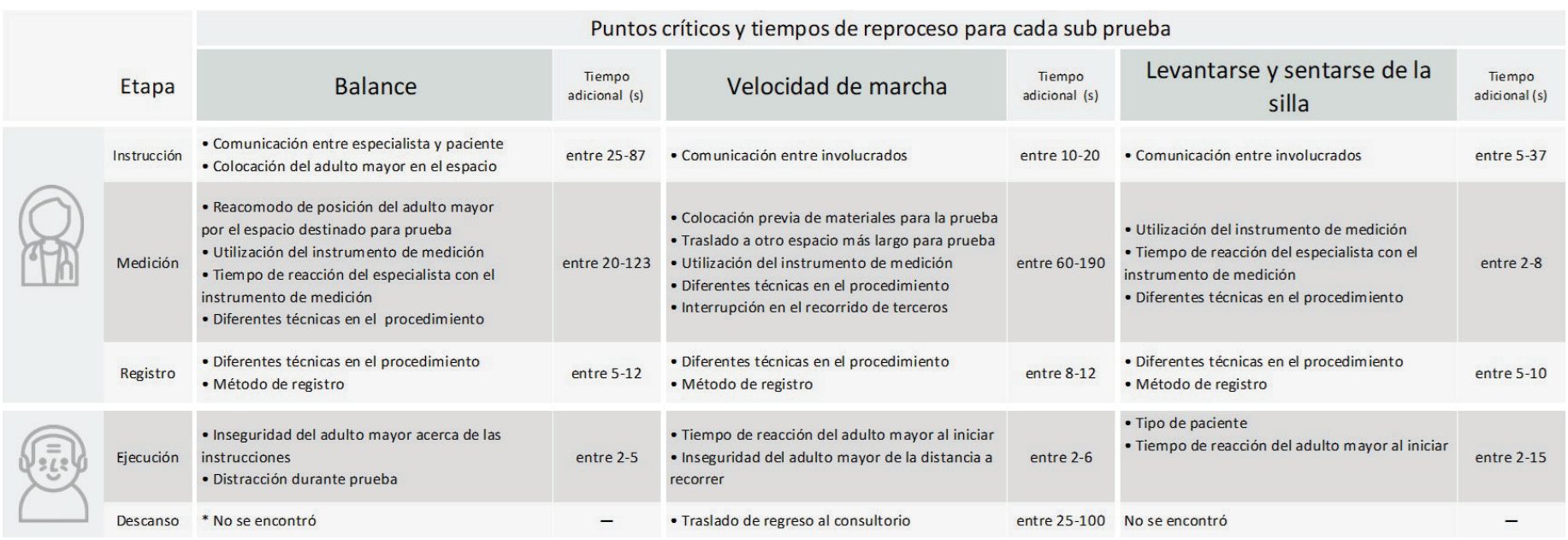

Y para la indicación de esta prueba, nuevamente pacientes que dudaban de las indicaciones provocaban que el especialista demostrara e hiciera todo el recorrido ida y vuelta (de 4 metros de largo). En la prueba de levantarse de la silla, se realiza una pre prueba para que conocer si el adulto mayor es capaz de sentarse y levantarse de la silla. Esta prueba se realiza 1 vez, sin embargo, el paciente con trastorno cognitivo y el que utiliza una andadera siguieron realizando la prueba una vez iniciaron el movimiento, con lo que se tuvo que detener la prueba y tomar un descanso de por lo menos un minuto.

Una vez realizado el proceso de identificación de los puntos críticos, tipos de reproceso e influencia de las características del paciente para cada prueba, se definieron cuáles causas podrían estar afectando la fiabilidad de la medición en la prueba SPPB, así como el tiempo para aplicarla. Dichos factores fueron apoyados también por las necesidades específicas del especialista y el adulto mayor en la prueba. Se ordenaron por grupos de necesidades y se establecieron catorce factores directos e indirectos (cuadro 3). Los factores que los especialistas consideraron más críticos fueron relacionados al instrumento de medición. La fisioterapeuta usaba un cronómetro en el reloj digital de pulsera mientras que el médico geriatra utilizó el celular. Mientras el médico sentía incomodidad por prestar atención a la prueba y manejar el celular, la fisioterapeuta expresó la imposibilidad de reaccionar con rapidez, por finalizar el cronómetro, en situaciones donde el adulto mayor puede desbalancearse. Otra observación importante se dio en el tipo de procedimiento. Mientras que la fisioterapeuta registraba todos los datos al final de cada subprueba, el geriatra lo hacía después de cada posición o movimiento 
con lo que existían más pausas. Ambos especialistas coincidieron en la dificultad de aplicar la misma prueba a pacientes con condiciones más severas (cognitivas o físicas), y que sólo debería de prescindir de ella los pacientes con discapacidad física. Finalmente, otro factor de alta importancia fue el seccionamiento de los espacios para aplicar la prueba, pues la prueba de velocidad solo puede hacerse en espacios largos como un pasillo. Además, las colocaciones de marcas son alteradas por otras personas aumentando el tiempo de aplicación (cuadro 3).

Cuadro 3. Necesidades y factores o causas que afectan la fiabilidad de los datos y el tiempo de aplicación en la prueba.

\begin{tabular}{|c|c|c|}
\hline No. & Necesidad & Factor \\
\hline 1 & Organización del lugar de trabajo & \multirow{2}{*}{ Desorganización } \\
\hline 2 & Equipo de trabajo colaborativo & \\
\hline 3 & Comunicación eficaz en la prueba & Comunicaión inadecuada \\
\hline 4 & $\begin{array}{l}\text { Similitud en la manera de atención } \\
\text { al paciente }\end{array}$ & Tipo de entrenamiento \\
\hline 5 & $\begin{array}{c}\text { Know how de la aplicación de la } \\
\text { prueba }\end{array}$ & \multirow{2}{*}{ Experiencia del especialista } \\
\hline 6 & $\begin{array}{l}\text { Agilidad del especialista en la } \\
\text { prueba }\end{array}$ & \\
\hline 7 & $\begin{array}{l}\text { Prueba realizable para los pacientes } \\
\text { con características diferentes }\end{array}$ & \multirow{2}{*}{ Tipo de paciente } \\
\hline 8 & $\begin{array}{l}\text { Disposición del paciente para } \\
\text { realizar la prueba }\end{array}$ & \\
\hline 9 & $\begin{array}{l}\text { Similitud en la aplicación de la } \\
\text { prueba por parte de diferentes } \\
\text { especialistas }\end{array}$ & Tipo de procedimiento \\
\hline 10 & $\begin{array}{l}\text { Estandarizar la aplicación de la } \\
\text { prueba }\end{array}$ & Proceso no estandarizado \\
\hline 11 & $\begin{array}{l}\text { Registrar el puntaje lo más rápido } \\
\text { posible }\end{array}$ & \multirow{2}{*}{ Diseño de la prueba } \\
\hline 12 & $\begin{array}{c}\text { Calcular el puntaje lo más rápido } \\
\text { posible }\end{array}$ & \\
\hline 13 & $\begin{array}{l}\text { Instrumento de medición preciso y } \\
\text { fácil de usar }\end{array}$ & Tipo de tecnología \\
\hline 14 & Materiales de apoyo adecuados & \multirow[b]{2}{*}{ Tipo de material } \\
\hline 15 & $\begin{array}{l}\text { Colocación rápida y que no sea } \\
\text { manipulada por terceros }\end{array}$ & \\
\hline 16 & Materiales duraderos & Frecuencia alta de mantenimiento \\
\hline 17 & $\begin{array}{l}\text { Tiempo de aplicación de la prueba } \\
\text { rápido dentro de la consulta }\end{array}$ & Tiempo limitado \\
\hline 18 & $\begin{array}{c}\text { Marcar o delimitar el espacio para } \\
\text { las posiciones }\end{array}$ & Señalización inexistente \\
\hline 19 & $\begin{array}{l}\text { No seccionar la prueba en dos } \\
\text { espacios (para evitar traslados) }\end{array}$ & Espacio pequeño \\
\hline
\end{tabular}




\section{Conclusiones}

La prueba de valoración funcional SPPB tiene gran validez y frecuencia de uso a nivel internacional, con el fin de detectar de una manera rápida el estado de fragilidad del adulto mayor y predecir una discapacidad a largo plazo. Está hecha para visualizar tres capacidades en el adulto mayor: su equilibrio, su fuerza de piernas y su marcha en un tiempo determinado. Sin embargo, se han observado ciertos factores críticos que influyen en dicha medición y, por lo tanto, en el criterio final provocando desconfianza en los datos.

Los puntos más críticos se observaron en el manejo del instrumento de medición (un cronómetro), el manejo del espacio para aplicar la prueba y la manera en la que se dan las instrucciones. Esto afecta la fiabilidad de los datos y que el tiempo de aplicación aumente. Además, los reprocesos identificados se asocian con el tipo de paciente que realiza la prueba, la reacomodación del paciente para que vuelva a tomar la postura adecuada y vuelva a ser medido o la demostración de las instrucciones que ya se habían dado. Y, por último, los factores que más afectan son el tipo de tecnología, tipo de paciente, modo de aplicar la prueba y el espacio de aplicación. De acuerdo a este análisis se ve el potencial que tiene la incorporación de la tecnología en este tipo de pruebas de valoración, en donde se pueda apoyar la labor del especialista geriátrico y brindarle mayor confianza en sus mediciones y en la rehabilitación del paciente.

\section{Referencias}

[1] Ministerio de Planificación Nacional y Política Económica de Costa Rica, «COSTA RICA: Prospectiva en cambio demográfico al 2045, » San José, 2017.

[2] N. Eynon, C. Yamin, D. Ben-Sira y M. Sagiv, «Optimal health and function among the elderly: lessening severity of ADL disability, " European Review of Aging and Physical Activity, vol. 6, n 55, 2009.

[3] E. DENT, J. MORLEY, A. CRUZ-JENTOFT, L. WOODHOUSE, L. RODRÍGUEZ-MAÑAS y L. FRIED, «PHYSICAL FRAILTY: ICFSR INTERNATIONAL CLINICAL PRACTICE GUIDELINES FOR IDENTIFICATION AND MANAGEMENT, » The journal of nutrition, health \& aging, vol. 23(9), pp. 771-787, 2019.

[4] A. CRUZ-JENTOFT, G. BAHAT, J. BAUER, Y. BOIRIE, O. BUYÈRE y T. CEDERHOLM, «Sarcopenia: revised European consensus on definition and diagnosis, » Age and Aging, vol. 48 (1), pp. 16-31, 2019.

[5] F. MORALES M, «La Atención Integral De Las Personas Adultas Mayores En Costa Rica,» [En línea]. Available: http://www.apronage.or.cr/13-articulos-personal-de-salud/27-la-atencion-integral-de-las-personas-adultasmayores-en-costa-rica. [Último acceso: 2904 2019].

[6] J. Guralnik, E. Simonsick, L. Ferrucci, R. Glynn, L. Berkman, D. Blazer, P. Scherr y R. Wallace, «A short physical performance battery assessing lower extremity function: association with self-reported disability and prediction of mortality and nursing home admission,» Journal of Gerontology, vol. 49(2), pp. 85-94, 1994.

[7] Hospital Nacional de Geriatría y Gerontología Dr. Raúl Blanco Cervantes, PROTOCOLO DE LA PRUEBA CORTA DE DESEMPEÑO FÍSICO (SPPB) Y HOJA DE CALIFICACIÓN, San José, 2019.

[8] Sistema Costarricense de Información Jurídica, «Reglamento a la Ley Reguladora de Investigación Biomédica, NN 39061-S,» 2019. [En línea]. Available: http://www.pgrweb.go.cr/scij/Busqueda/Normativa/Normas/nrm_ texto_completo.aspx?param1=NRTC\&nValor1=1\&nValor2=79779\&nValor3=103451\&strTipM=TC.

[9] J. Guralnik, L. Ferrucci, E. Simonsick, S. M.E y ,. R. Wallace, «Lower-extremity function in persons over the age of 70 years as a predictor of subsequent disability, " The New England Journal of medicine, vol. 332, $\mathrm{n}^{\circ}$ 9, pp. 556-61, 1995.

[10] S. Perara, S. Mody, R. Woodman y S. Studenski, «Meaningful Change and Responsiveness in Common Physical Performance Measures in Older Adults, " The American Geriatrics Society , vol. 54, n 5, pp. 743-749, 2006.

[11] Ministerio de Sanidad, Servicios Sociales e Igualdad de España, «Documento de consenso sobre prevención de fragilidad y caídas en la persona mayor. Estrategia de Promoción de la Salud y Prevención en el SNS, » ESTILO ESTUGRAF-IMPRESORES, S.L, Madrid, 2014. 